\title{
Performance evaluation of two fundus oculi angiographic imaging system: Optos 200Tx and Heidelberg Spectralis
}

\author{
SHUANG LI, JING-JING WANG, HONG-YANG LI, WEI WANG, MENG TIAN, \\ XU-QIANG LANG and KANG WANG
}

Department of Ophthalmology, Beijing Friendship Hospital, Capital Medical University, Beijing 100050, P.R. China

Received December 15, 2018; Accepted October 29, 2019

DOI: $10.3892 / e t m .2020 .9451$

\begin{abstract}
The present study aimed to compare the imaging performance of two ultra-wide-field fluorescein angiography imaging systems, namely the OptosOptomap 200Tx (Optos 200Tx) and the Heidelberg Spectralis (Spectralis). A total of 18 patients (36 eyes) underwent angiography using the two systems at the Department of Ophthalmology, Beijing Friendship Hospital (Beijing, China) between January and June 2017. The images were obtained as a single shot centered on the macula. The total area and area within each of four visualized quadrants were calculated and compared. The averages of the total and individual quadrant area captured by the Optos 200Tx were all larger than those obtained with the Spectralis $(\mathrm{P}<0.05)$. For pair-wise comparison, the circular area centered on the macula (radius of 10 and $15 \mathrm{~mm}$ ) was displayed: Optos 200Tx $10 \mathrm{~mm}$ $\left(295.57 \mathrm{~mm}^{2}\right)<$ Spectralis $\left(520.11 \mathrm{~mm}^{2}\right)<$ Optos 200Tx $15 \mathrm{~mm}$ $\left(596.45 \mathrm{~mm}^{2}\right)<$ Optos 200Tx $\left(804.36 \mathrm{~mm}^{2}\right)(\mathrm{P}<0.01)$. The differences of each of the four quadrant areas were statistically significant between the two systems $(\mathrm{P}<0.05)$. The mean size of the areas was in the following order: Inferior $<$ temporal $<$ superior < nasal for the Optos 200Tx, and inferior < temporal $<$ nasal $<$ superior for the Spectralis. Further comparison of the four-quadrant area indicated that the inferior quadrant of the Optos 200Tx was smaller than the other three quadrants $(\mathrm{P}<0.01)$ and the inferior quadrant of the Spectralis was smaller than the superior quadrant $(\mathrm{P}<0.01)$. The total retinal area and the retinal area of each quadrant captured by the Optos 200Tx were larger than those captured with the Spectralis. The total retinal area captured with the Optos 200Tx was able to cover the mid-peripheral area and part of the far-peripheral area of the retina, whereas the Spectralis only covered the mid-peripheral area.
\end{abstract}

Correspondence to: Dr Kang Wang, Department of Ophthalmology, Beijing Friendship Hospital, Capital Medical University, 95 Yongan Road, Xicheng, Beijing 100050, P.R. China E-mail: wangkang0802@163.com

Key words: ultra-wide-angle imaging, fundus fluorescein angiography, Optos 200Tx, Heidelberg Spectralis

\section{Introduction}

Ultra-wide-field fluorescein angiography (UWFA) is a novel technology that has developed rapidly in recent years $(1,2)$. As numerous pathological changes of fundus diseases occur at the edge of the retina, the limitation of imaging leads to insufficient diagnosis or underestimation of the severity of the disease $(3,4)$. Therefore, clear imaging of the edge of the retina is important for the diagnosis, monitoring and prognostication of patients with ocular fundus diseases. The traditional fundus fluorescein angiography system may only provide a vision field ranging from 30 to 55 degrees (5). Even with the conventional 7 standard field imaging proposed by the Early Treatment Diabetic Retinopathy Study, it may only cover the 75-degree fields, which accounts for $\sim 25 \%$ of the total area of the retina. Thus, it cannot fully display the retinopathy $(6,7)$. By contrast, the current ultra-wide-angle technology has been able to provide a vision ranging from 100 to 200 degrees (8-10). In addition, by using the traditional fundus fluorescein angiography system, observation and precise measure of structures and lesions around the edge of the fundus may only be performed through later image reconstruction (11). However, the blood circulation is a dynamic process and there is a time delay in the images captured; therefore, there are certain limitations in the observation of the structure of the retina and choroid, as well as the blood circulation.

The current UWFA technology features ultra-wide-angle color photography, autofluorescence imaging and fluorescein angiography, and all of these have been widely used in the observation and research of marginal retinal diseases $(1,12)$. However, clinical data produced using this imaging technology are still limited. By collecting clinical research data, the advantages and disadvantages of UWFA technology may be elucidated, which is key in the interpretation of images and the application of UWFA may be constantly enhanced. This will facilitate the accuracy of the assessment of fundus diseases, even under complex conditions.

At present, the most widely used UWFA devices on the market are the 200Tx Optos ultra-widefield imaging system (Optus) and the Spectralis HRA+ optical coherence tomography (OCT) system (Heidelberg Spectralis) $(13,14)$. These two types of ultra-wide-angle fundus imaging device may obtain retinal images in a variety of modes of the posterior pole and the marginal region through non-contact single photographing 
without any later image reconstruction $(1,15)$. However, there are certain different characteristics in imaging between the two systems. On a single non-steered image, the Optos covers a larger total retinal area and greater image variability than the Spectralis, while the Spectralis captures the superior and inferior retinal vasculature more peripherally (14). To date, no comprehensive comparative analysis of the two imaging systems has been performed. In the present study, macula centered images obtained from a single shot by using the two devices, Optos and Spectralis, were analyzed (Fig. 1). The total area of the two UWFA images, as well as the retina imaging area of the four quadrants, which are nasal, superior, inferior and temporal, were compared. The present study provides practical information for the clinical application of these two systems.

\section{Patients and methods}

Study design and participants. A single-center, open-labeled prospective cohort study was performed at the Department of Ophthalmology, Beijing Friendship Hospital, Capital Medical University (Beijing, China) from January to June 2017. A total of 18 adult patients (36 eyes) were screened for inclusion. The inclusion criteria were as follows: Age, $\geq 18$ years; diopter interstitial clear; best-corrected visual acuity $>20 / 200$. The following exclusion criteria were applied: The eye condition changed significantly during the first 3 months after the first fundus examination; retinal detachment and choroidal detachment; difficult image acquisition due to poor vision; intolerance or poor cooperation with fluorescein fundus angiography (FFA) examination; allergy to fluorescein sodium (for injection).

Fluorescein angiography and image selection. For outcome evaluation, the total area and area within each of four visualized quadrants (superior, inferior, nasal and temporal) were calculated and compared. The 200Tx Optos ultra-wide field imaging system (Optus) and the Spectralis HRA+OCT system (Heidelberg Spectralis) were used to collect fundus angiography images.

First, allergy to the contrast media was tested by a subcutaneous test; a specialized technician dilated the pupil for half an hour by using tropicamide phenylephrine eye drops. Subsequently, $1 \%$ fluorescein sodium diluent was injected and observed to confirm that the patients were not allergic to the contrast media. Subsequently, $5 \mathrm{ml} 20 \%$ fluorescein sodium injection was injected in $5 \mathrm{sec}$ via the elbow vein. For the Spectralis, after capturing a 30 -sec video by using a 102 degrees super wide-angle front lens, the patients were instructed to rotate their eyeballs in 9 directions in turn: The posterior pole, superior, superior temporal, temporal, infratemporal, inferior, superior nasal, nasal and infra nasal direction, and 1 image was collected per second. For the Optos 200Tx, after capturing 60-sec images of the posterior pole, the patients were instructed to rotate their eyeballs in 4 directions in turn: Superior, inferior, nasal and temporal, and 1 image was collected per second (Fig. 1). The retinogram images of $30 \mathrm{sec}$ to $3 \mathrm{~min}$, clear, representative and venous phase, were selected for comparative analysis. All of the angiographic images were captured by an experienced technician. UWFFA images from the Optos 200Tx were transformed to stereographic projection images using proprietary software available from the manufacturer (Doheny Eye Institute) before quantitative analysis (16).

Quantitative analysis method and quality control of FFA images. First, two different modal images of the Spectralis and Optos 200Tx were calibrated and standardized by using Image J 1.49b software (National Institutes of Health). The retina profiles of the FFA images captured by the two devices were then respectively depicted by using Adobe ${ }^{\circledR}$ Photoshop CS6 (Adobe Inc.). Grading results were saved as binary masks and subsequently calculated in square millimeters by summing the size of all pixels that make up the mask using the manufacturer's (16) quantification software (Doheny Eye Institute). If the UWFA image was poor, the image quality was improved by adjusting the contrast and brightness, and subsequently, the contour of the image was accurately delineated and measured. The analysis regions included the following: a) Centered on the macula, the area was evenly divided into four quadrants: Superior, inferior, nasal and temporal; b) centered on the macula, areas with a diameter of 10 and $15 \mathrm{~mm}$. The drawing process is illustrated in Fig. 2 and 3. The total area and the four quadrant areas of the contrast images captured with the two devices were individually calculated. The total area $\left(\mathrm{mm}^{2}\right)$ of FFA retinal imaging and the captured area of the regions from the two devices were compared.

Statistical analysis. SPSS 20.0 software (IBM Corp.) was used for statistical analysis of the data. Measurement data were expressed as the mean \pm standard deviation. For comparison between different devices in the same test area, a paired Student's t-test was used when data conformed to a normal distribution and Wilcoxon's signed-rank test was used when data did not conform to a normal distribution. The Shapiro-Wilk method was used to test the normality of distribution of the data. The Friedman test was used to assess differences between directions with the same instrument, and post-hoc analysis with a paired Student's t-test or Wilcoxon signed-rank tests was performed with a Bonferroni correction applied. All tests were bilateral and $\mathrm{P}<0.05$ was considered to indicate statistical significance.

\section{Results}

Characteristics of the participants. A total of 72 FFA images from 18 patients (36 eyes) were included in the present study (Table I), including 11 male cases with 22 eyes and 7 female cases with 14 eyes. The patients' age ranged from 53 to 82 years and the average age was $63.80 \pm 12.50$ years. The diseases included diabetic retinopathy (DR; 18 eyes), central retinal vein occlusion (3 eyes), retinal branch vein occlusion (3 eyes), wet age-associated macular degeneration (1 eye), anterior ischemic optic neuropathy (1 eye), ocular ischemic syndrome (1 eye) and retina lattice degeneration (1 eye), as well as healthy eyes $(n=8)$. All subjects were examined with the Spectralis equipment first and then with the Optos 200Tx after a period of time. The interval of the two examinations was $<3$ months. In addition, a paired comparison of two images obtained from the same eye of the same patient was performed. 


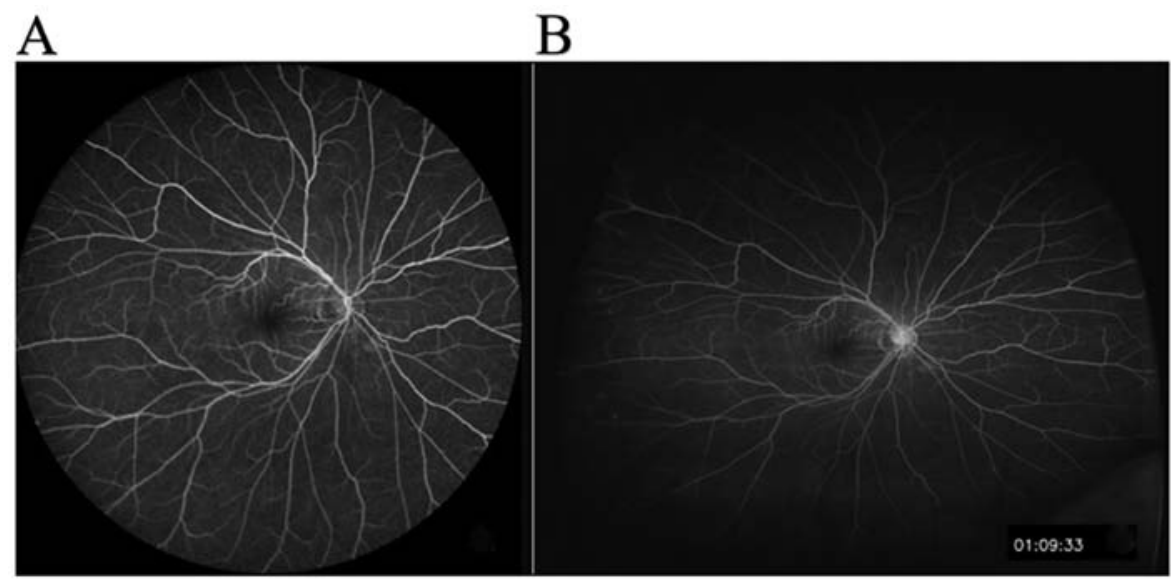

Figure 1. Venography image of single shot with the macula as the center using (A) the Spectralis or (B) the Optos 200Tx system.
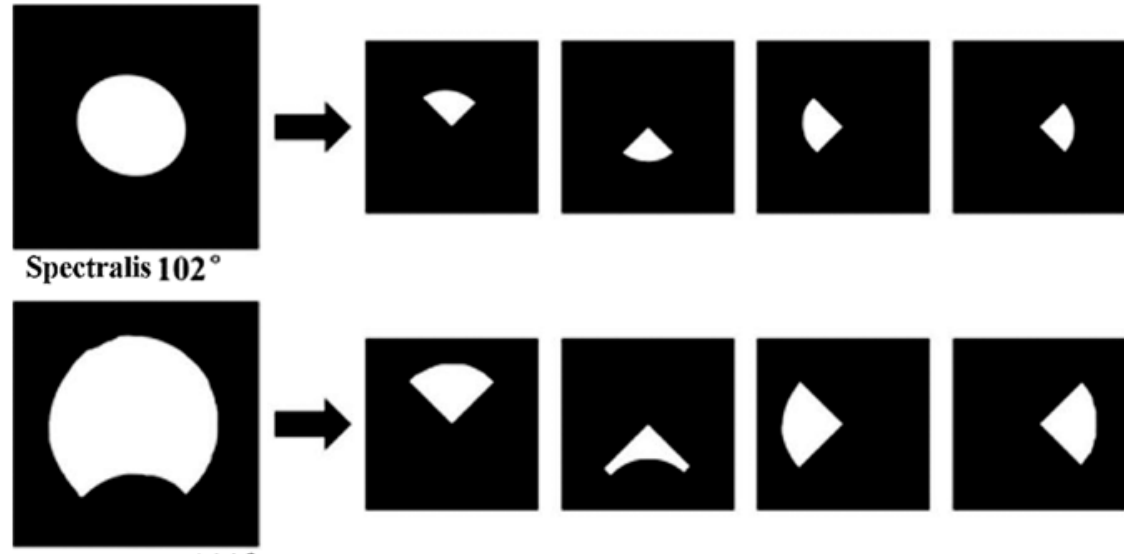

Optos 200Tx 200

Figure 2. The 3D-transferred contrast images of the Spectralis and Optos 200Tx after standardization. The four quadrants of the superior, temporal, inferior and nasal regions were classified with macula as the center. After the transformation into gray scale format image via Adobe Photoshop CS6, automatic measurement of the area of the target retina was performed.

Table I. Demographic characteristics of the patients $(\mathrm{n}=18)$.

\begin{tabular}{lc}
\hline Item & Value \\
\hline Age (years) & $63.80 \pm 12.50$ \\
Sex (male/female) & $11 / 7$ \\
Disease type (eye/eyes) & \\
Diabetic retinopathy & 18 \\
Central retinal vein occlusion & 3 \\
Retinal branch vein occlusion & 3 \\
Wet age-related macular degeneration & 1 \\
Anterior ischemic optic neuropathy & 1 \\
Ocular ischemic syndrome & 1 \\
Retina lattice degeneration & 1 \\
Healthy & 8 \\
\hline
\end{tabular}

Data were expressed as mean \pm standard deviation.

Analysis of accurate measurement results of the FFA imaging area. The total FFA retina imaging areas and the areas of the two regions within the radius of the Spectralis and Optos 200Tx are provided in Table II. The areas exhibited the following trend: Spectralis $10 \mathrm{~mm}=$ Optos 200Tx $10 \mathrm{~mm}<$ Spectralis $15 \mathrm{~mm}<$ Spectralis total < Optos 200Tx $15 \mathrm{~mm}<$ Optos 200Tx total. The results suggested that there was no statistically significant difference between total area and $15-\mathrm{mm}$ area of the Spectralis $(\mathrm{P}>0.05)$, while the other differences in the pairwise comparisons were statistically significant $(\mathrm{P}<0.05)$.

Analysis of area measurement results of FFA quadrant imaging. A qualitative comparison of the retinal imaging area in the four quadrants of the superior, temporal, inferior and nasal field between the Spectralis and Optos 200Tx is provided in Fig. 2, and an intra-group comparison of the four quadrants is also displayed in Fig. 2. The four quadrant imaging areas of the Optos 200Tx FFA were larger than those of the Spectralis and the difference was statistically significant. The inter-group comparison of the four quadrant imaging areas of the Spectralis and Optos 200Tx revealed statistically significant differences $(\mathrm{P}<0.001)$. The further analysis indicated that the order of the mean area was as follows: For the Spectralis, inferior < temporal < nasal < superior; for the Optos 200Tx, inferior $<$ temporal $<$ superior $<$ nasal $($ Table III). The 
Table II. Comparison of FFA imaging total area between the Spectralis and Optos 200Tx.

\begin{tabular}{lcrr}
\hline Imaging method & Optos 200Tx $\left(\mathrm{mm}^{2}\right)$ & Heidelberg Spectralis $\left(\mathrm{mm}^{2}\right)$ & Statistic $(\mathrm{t} / \mathrm{S})$ \\
\hline FFA imaging total & $804.36 \pm 68.61$ & $520.11 \pm 32.77$ & -25.939 \\
15 mm region & $596.45 \pm 33.24$ & $509.2 \pm 49.13$ & 12.676 \\
10 mm region & $295.57 \pm 2.93$ & $291.79 \pm 17.68$ & 5.000 \\
\hline
\end{tabular}

Data were expressed as mean \pm standard deviation. The statistics of $t$ and $S$ are corresponding to a Paired Student's t-test and Wilcoxon sign-rank test, respectively. FFA, fluorescein fundus angiography.

Table III. Inter-group comparison of four quadrant imaging areas of fluorescein fundus angiography.

\begin{tabular}{lcrr}
\hline Imaging orientation & Optos 200Tx $\left(\mathrm{mm}^{2}\right)$ & Heidelberg Spectralis $\left(\mathrm{mm}^{2}\right)$ & Statistic(t/S) \\
\hline Superior & $207.08 \pm 36.41$ & $142.95 \pm 48.12$ & -294.000 \\
Inferior & $162.21 \pm 33.56$ & $124.4 \pm 11.42$ & -328.000 \\
Nasal & $226.11 \pm 22.18$ & $133.2 \pm 14.2$ & -333.000 \\
Temporal & $204.27 \pm 33.12$ & $127.43 \pm 19.94$ & -20.039 \\
\hline
\end{tabular}

Data were expressed as mean \pm standard deviation. The statistics of $\mathrm{t}$ and $\mathrm{S}$ are corresponding to a Paired Student's $\mathrm{t}$-test and Wilcoxon signrank test, respectively.

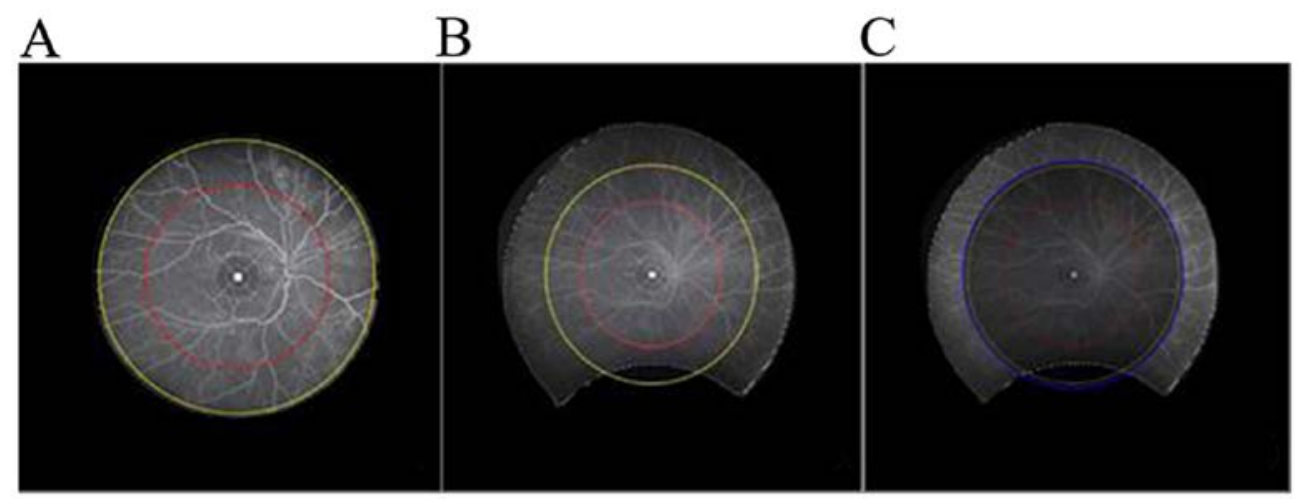

Figure 3. Contrast images single shot with the macula as the center. Images of the same eye taken by (A) the Spectralis and (B) the Optos 200Tx. (C) Overlap of the two images in A and B. The white spot is the macular fovea. The red and yellow circles are circles with the macular as the center and a radius of 10 and $15 \mathrm{~mm}$, respectively. The blue circle indicates the contrast range of the Spectralis. The white dotted line in A is the retina imaging boundary of the Spectralis and the white dotted lines in $\mathrm{B}$ and $\mathrm{C}$ are the retina imaging boundary of the Optos 200Tx.

differences in retinal imaging area between the inferior quadrant and the superior quadrant of the Spectralis FFA image were statistically significant (Bonferroni-adjusted $\mathrm{P}<0.05$ ), while the remaining pairwise comparisons revealed no significant difference (Bonferroni-adjusted $\mathrm{P}>0.05$ ). Regarding the area of the Optos 200Tx FFA retina imaging, the comparison between the temporal and the superior quadrant indicated no statistically significant difference (Bonferroni-adjusted $\mathrm{P}>0.05$ ), while the results of the pairwise comparisons of other quadrants revealed statistically significant differences (Bonferroni-adjusted $\mathrm{P}<0.05$ ). The intra-group comparison for the Optos 200Tx (Friedman test, $\mathrm{P}<0.001$ ) and the Spectralis (Friedman test, $\mathrm{P}=0.026$ ) is provided in Tables IV and $\mathrm{V}$, respectively.

For the FFA macular-centered single-shot images that were used to detect peripheral retinal hiatus, vascular leakage, neovascularization and no perfusion area, the Optos 200Tx was less likely to miss anomalies compared with the Spectralis at 55 and 102 degrees. Furthermore, the lesions may be treated by using a laser or other methods under the guidance of the Optos 200Tx FFA, as presented in Fig. 4.

In the representative images in Fig. 5, the images were taken for an FFA test, as a single shot with the macula as the center and the patient had diabetes for 10 years. A microangioma was detected in the inferior field using the Spectralis at 102 degrees, but nothing was detected using the Optos 200Tx.

\section{Discussion}

UWFA is a novel technology for retina examination, but its clinical application of it is not very extensive (17), as clinicians are currently not very familiar with it. Elucidation of 

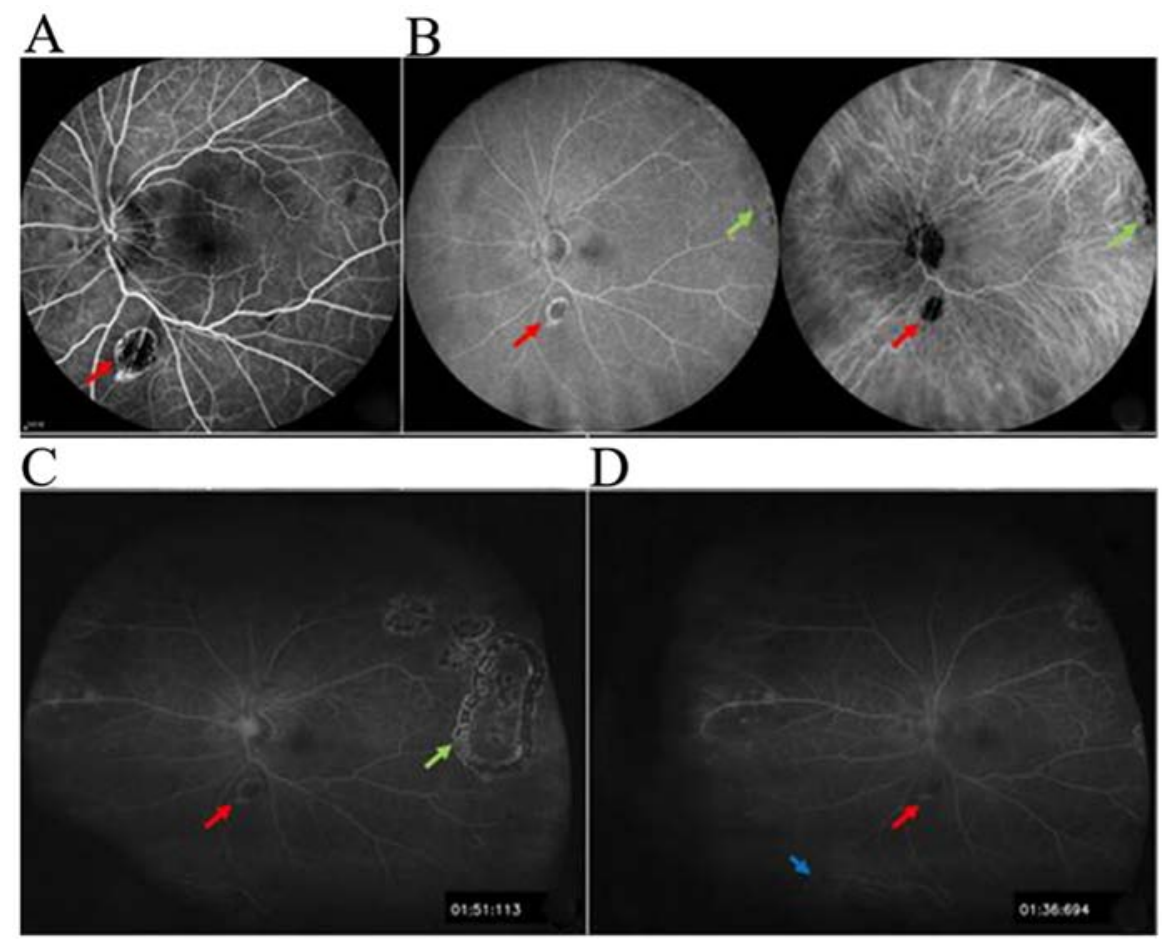

Figure 4. (A) Image captured with the Spectralis 55 degrees. (B) Image taken with the Spectralis 102 degrees + ICGA. (C) FFA image captured with the Optos 200Tx with the macula as the center. (D) FFA image taken with the Optos 200Tx and the patient looking down to the right. The choroidal defect lesions (red arrowheads) may be displayed with the Spectralis as well as with the Optos 200Tx, while the resolution and contrast of the Spectralis are better than those of the Optos 200Tx. The green arrows in B and C and the blue arrow in D point to the lattice-like degeneration area of the peripheral retinal holes after laser closure. The lattice-like degeneration area, which cannot be completely displayed by Spectralis 102 degrees FFA + ICGA imaging in B, is fully displayed on the Optos 200Tx FFA image in C. The inferior peripheral retinal degeneration area that cannot be displayed in C is captured when the patient looks at the lower right side. ICGA, indocyanine green angiography; FFA, fluorescein fundus angiography.

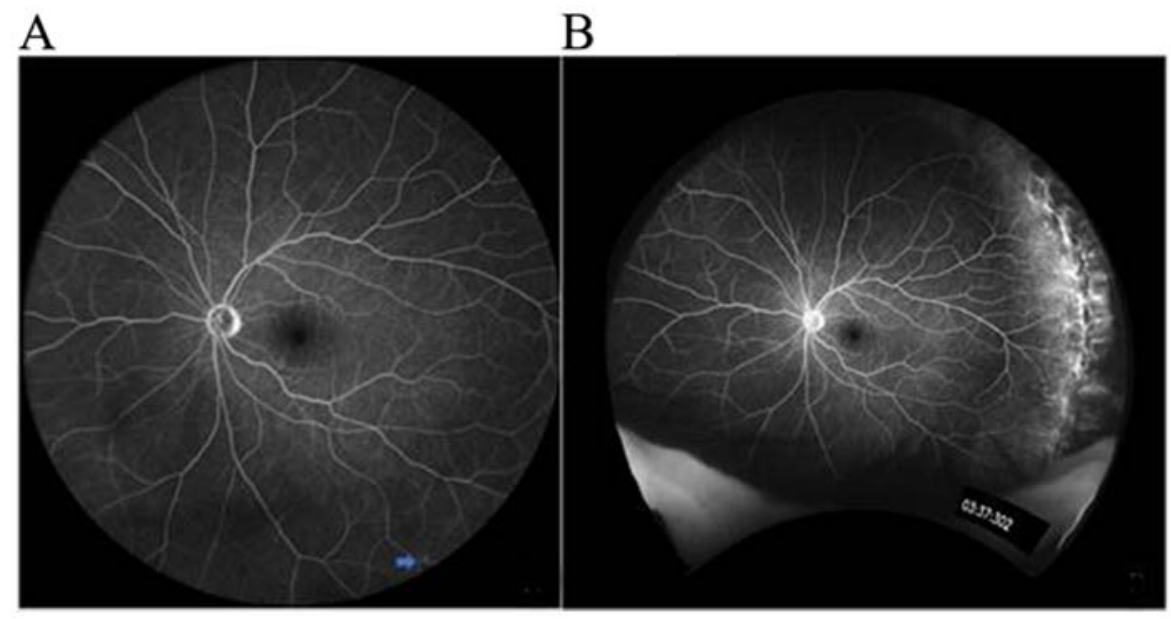

Figure 5. Fluorescein fundus angiography images of the same eye with amicroangioma. (A) Image captured with the Spectralis 102 degree; the blue arrow indicates microangioma. (B) Image captured with the Optos 200Tx 200 degrees; the microangioma lesion was not displayed, while the mottled fluorescence in the peripheral temporal area of the retina is visible.

the advantages and disadvantages of UWFA technology may allow for continuous enhancement of the clinical application of UWFA and may lead to an improved understanding of fundus diseases. The Optos 200Tx and Spectralis are the two most widely used devices in the clinic and the present study compared the imaging scope and characteristics of the two devices.

In the present study, the total imaging area and the imaging area of the four quadrants, namely superior, temporal, inferior and nasal, of the two devices were first compared. The results indicated that the total FFA imaging area and the area of the four quadrants of the Optos 200Tx were significantly larger than those of the Spectralis.

In addition, intra-group comparisons of the four quadrant imaging areas (superior, temporal, inferior and nasal) of the two sets of equipment were performed in the present study. The results indicated that in the FFA images obtained from a macular-centered single shot, the inferior quadrant was the 
Table IV. Comparison of different imaging positions of the Optos 200Tx.

\begin{tabular}{lrrr}
\hline Imaging orientation & $\begin{array}{c}\text { Difference } \\
\text { value }\left(\mathrm{mm}^{2}\right)\end{array}$ & \multicolumn{1}{c}{$\begin{array}{c}\text { Statistic } \\
(\mathrm{t} / \mathrm{S})\end{array}$} & P-value \\
\hline Superior vs. inferior & $44.87 \pm 56.41$ & 261.000 & $<0.001$ \\
Superior vs. nasal & $-19.02 \pm 37.92$ & -210.000 & $<0.001$ \\
Superior vs. temporal & $2.81 \pm 37.75$ & 0.447 & 0.658 \\
Inferior vs. nasal & $-63.9 \pm 37.92$ & -330.000 & $<0.001$ \\
Inferior vs. temporal & $-42.06 \pm 42.64$ & -286.000 & $<0.001$ \\
Nasal vs. temporal & $21.84 \pm 44.95$ & 157.000 & 0.008 \\
\hline
\end{tabular}

Data were expressed as mean \pm standard deviation. The statistics of $\mathrm{t}$ and $\mathrm{S}$ are corresponding to a Paired Student's t-test and Wilcoxon sign-rank test, respectively.

smallest, while the superior and the nasal quadrants were relatively large. It was speculated that this may be due to the eyelash occlusion in the inferior quadrant and the contraction of the orbicularis oculi muscle caused by strong light, while the upper eyelid is relatively relaxed and easy to move away, so the superior quadrant imaging is less affected. The comparison indicated that there was almost no significant difference in the imaging scope of the four quadrant radiography images of the Spectralis; only the difference between the superior and inferior was statistically significant. The possible reasons why the inferior quadrant imaging area was less than that of the superior quadrant were chiefly as follows: First, the small sample size may have led to a larger class I error; furthermore, all image peripheral deformations were corrected based on the Optos 3D first and the area was then measured and this may have introduced certain errors to the area measurement with the Spectralis. For the LSD analysis of the imaging area of quadrants of the Optos 200Tx, the pairwise comparisons revealed statistically significant differences except for superior vs. temporal. The nasal and temporal imaging areas were large and the superior and inferior areas were insufficient. The study by Witmer et al (14) from 2013 indicated that the retinal vascular imaging ranges of the nasal and temporal region of the Optos 200Tx were closer to the edge than those of the Spectralis, while the opposite was revealed for the superior and inferior fields in the same aforementioned study. In the present study, for the imaging area of each quadrant of the two systems, the inferior field was the smallest, followed by the temporal field. The Spectralis displayed the peripheral retina structure more completely in the superior field, while the Optos 200Tx better displayed the nasal and temporal areas. This was highly consistent with the conclusions of Witmer et al (14). In addition, in the present study, the images of the superior and inferior quadrants of the Optos 200Tx were frequently obscured by artifacts, eyelashes and eyelids.

Widmer et al (18) divided the retina into a middle round, a ring-shaped area with a radius of $10-15 \mathrm{~mm}$, and a distal round, which is the peripheral ring-shaped area of the retina with a radius of $>15 \mathrm{~mm}$. In the present study, the imaging area of the FFA images centered on the macula and radii of 10 and $15 \mathrm{~mm}$ was measured with the two sets of devices and
Table V. Comparison of different imaging positions of the Heidelberg Spectralis.

\begin{tabular}{lrrr}
\hline Imaging orientation & $\begin{array}{c}\text { Difference } \\
\text { value }\left(\mathrm{mm}^{2}\right)\end{array}$ & \multicolumn{1}{c}{$\begin{array}{c}\text { Statistic } \\
(\mathrm{t} / \mathrm{S})\end{array}$} & P-value \\
\hline Superior vs. inferior & $18.55 \pm 56.09$ & 203.000 & 0.001 \\
Superior vs. nasal & $9.75 \pm 48.96$ & 80.000 & 0.213 \\
Superior vs. temporal & $15.51 \pm 59.35$ & 126.000 & 0.046 \\
Inferior vs. nasal & $-8.8 \pm 19.48$ & -140.000 & 0.026 \\
Inferior vs. temporal & $-3.03 \pm 16.37$ & -1.111 & 0.274 \\
Nasal vs. temporal & $5.77 \pm 27.79$ & 10.000 & 0.878 \\
\hline
\end{tabular}

Data were expressed as mean \pm standard deviation. The statistics of $\mathrm{t}$ and $\mathrm{S}$ are corresponding to a Paired Student's t-test and Wilcoxon sign-rank test, respectively.

compared. The areas exhibited the following trend: Spectralis $10 \mathrm{~mm}=$ Optos 200Tx $10 \mathrm{~mm}<$ Spectralis $15 \mathrm{~mm}<$ Spectralis total < Optos 200Tx $15 \mathrm{~mm}<$ Optos 200Tx total, and the differences were statistically significant. The further analysis indicated that the difference of the FFA area between the total and $15 \mathrm{~mm}$ of the Spectralis was not statistically significant, but the other pairwise comparisons revealed significant differences. The overall FFA imaging area of the Optos 200Tx was the largest and the imaging radius was $>15 \mathrm{~mm}$. This means that the Optos 200Tx is able to cover the distal part of the retina, which may display pathological changes of common fundus diseases. The FFA imaging radius of the Spectralis was close to or slightly longer than $15 \mathrm{~mm}$, which means the coverage of the Spectralis reached the middle retina and failed to cover the far periphery. Therefore, the images taken with the Spectralis system did not fully display any fundus lesions in the distal round and the more peripheral areas, but this may be compensated by the later image mosaicking.

The total area and the area of the four quadrants obtained by FFA retinal imaging with the Optos 200Tx were larger than those of the Spectralis and the total area exceeded the radius of $15 \mathrm{~mm}$. These results indicate that the imaging range of the Optos 200Tx is better than that of the Spectralis. Although the results of the present study suggest that the above two ultra-wide-angle equipment provide a comprehensive display of fundus lesions, including no perfusion area, microangioma, vascular leakage, neovascularization and retinal degeneration, when it comes to the peripheral retina, the Optos 200Tx was indicated to be more capable of displaying the above-mentioned fundus lesions (19). The Optos 200Tx is able to identify peripheral retinal lesions that are missed by the Spectralis, which is of great value for the guidance of clinical treatments, including laser therapy and intravitreal injection (19). This is of great significance for the monitoring of ocular fundus diseases, e.g. $\mathrm{DR}$, middle and anterior uveitis and retinal vasculitis, which frequently involve the distal peripheral retina.

The total imaging area of the Spectralis system was slightly smaller than that of the Optos 200Tx and the radius of the coverage region was between 10 and $15 \mathrm{~mm}$. Although its visible range is slightly smaller than that of the Optos 200Tx and has certain limitations in the observation of 
distal peripheral fundus lesions, the resolution of the built-in 55-degree lens and the front 102-degree ultra-wide-angle lens were better than those of the Optos 200Tx. Thus, small lesions may be clearly displayed with the Spectralis and it is able to detect microlesions of the macular region and the leakage of small-branch vessels, including macular edema and macular microangioma (20). The 102-degree ultra-wide-angle lens of the Spectralis combined with full mydriasis and rotation of the eye to different directions, as well as the later image mosaicking, extend the observation area to the near serrated edge (21). The combined application of the high-resolution 55-degree visual field imaging and the ultra-wide-angle lens may potentiate the comprehensive and thorough observation of minor lesions in key areas, including the peripheral retinopathy and the macular region of the posterior pole, and is able to reduce the missed diagnosis rate of the focal area. This will facilitate the diagnosis and treatment guidance of fundus diseases, including DR and isolated retinal vasculitis. These compensate the shortage of range of Spectralis imaging. The lesion of DR frequently occurs in the periphery of the retina, while lesions of retinal vein occlusion frequently appear on the posterior pole, near the periphery and around the middle part (22). In clinical practice, suitable equipment should be selected according to the imaging characteristics of UWFA equipment and the characteristics of the disease.

There are certain drawbacks of the present study that should be addressed: First, the sample size in the present study was relatively small and this is also the major limitation of the present study. Due to time constraints and difficult sample acquisition conditions, 18 cases were finally included. Compared with a previous study (14), in which only 5 patients with 10 eyes underwent ultra-widefield fluorescein angiography using the Optos ${ }^{\circledR}$ panoramic P200Tx imaging system and the non-contact ultra-widefield module in the Heidelberg Spectralis ${ }^{\circledR}$ HRA+OCT system, the results were similar with those of the current study. Furthermore, the images taken with the Optos 200Tx and Spectralis were not obtained simultaneously. Finally, due to time constraints and the small number of patients in line with the inclusion criteria, measurement of the non-perfusion area and assessment of the influence of different imaging ranges of the two UWFA devices on the ischemic index were not performed. The analysis results and methodology in the present study may help enhance the extent of clinical application of UWFA technology. In the future, further cases will be collected to reach a larger sample size and a more in-depth comparative analysis will be performed.

\section{Acknowledgements}

Not applicable.

\section{Funding}

This work was supported by grants from the High-level Health Technology Talents Training Program of the Beijing Health System and Research Foundation of Beijing Friendship Hospital, Capital Medical University (grant no. BJ-LM2017005L). The sponsor or funding organization had no role in the design or performance of this study.

\section{Availability of data and materials}

The datasets used and/or analyzed during the current study are available from the corresponding author on reasonable request.

\section{Authors' contributions}

SL and KW conceived and designed the experiments. SL, JJW, HYL, WW, MT and XQL performed the experiments. SL and JJW analyzed the data. SL wrote the paper. KW finally revised the paper. All authors had reviewed and agreed on the contents of this paper.

\section{Ethics approval and consent to participate}

The present study was approved by the Ethics Committee of the Affiliated Beijing Friendship Hospital, Capital Medical University (Beijing, China), and all procedures were designed and performed according to the Declaration of Helsinki. All patients were informed of the purpose and methods in detail prior to enrolment in the study and provided written informed consent to participate in the study.

\section{Patient consent for publication}

All patients consented for the publication of the data and photos in the current paper.

\section{Competing interests}

The authors declare that they have no competing interests.

\section{References}

1. Shoughy SS, Arevalo JF and Kozak I: Update on wide- and ultra-widefield retinal imaging. Indian J Ophthalmol 63: 575-581, 2015.

2. Calvo CM and Hartnett ME: The utility of ultra-widefield fluorescein angiography in pediatric retinal diseases. Int J Retina Vitreous 4: 21, 2018

3. Lim LS, Mitchell P, Seddon JM, Holz FG and Wong TY: Age-related macular degeneration. Lancet 379: 1728-1738, 2012.

4. Chen JJ and Kardon RH: Avoiding clinical misinterpretation and artifacts of optical coherence tomography analysis of the optic nerve, retinal nerve fiber layer, and ganglion cell layer. J Neuroophthalmol 36: 417-438, 2016.

5. Polans J, Keller B, Carrasco-Zevallos OM, LaRocca F, Cole E, Whitson HE, Lad EM, Farsiu S and Izatt JA: Wide-field retinal optical coherence tomography with wavefront sensorless adaptive optics for enhanced imaging of targeted regions. Biomed Opt Express 8: 16-37, 2016.

6. Rasmussen ML, Broe R, Frydkjaer-Olsen U, Olsen BS, Mortensen HB, Peto T and Grauslund J: Comparison between early treatment diabetic retinopathy study 7 -field retinal photos and non-mydriatic, mydriatic and mydriatic steered widefield scanning laser ophthalmoscopy for assessment of diabetic retinopathy. J Diabetes Complications 29: 99-104, 2015.

7. Gardner TW, Sander B, Larsen ML, Kunselman A, Tenhave T, Lund-Andersen H, Reimers J, Hubbard L, Blankenship GW, Quillen DA, et al: An extension of the Early Treatment Diabetic Retinopathy Study (ETDRS) system for grading of diabetic macular edema in the Astemizole Retinopathy Trial. Curr Eye Res 31: 535-547, 2006.

8. Witmer MT and Kiss S: Wide-field imaging of the retina. Surv Ophthalmol 58: 143-154, 2013.

9. Shifera AS, Pennesi ME, Yang P and Lin P: Ultra-wide-field fundus autofluorescence findings in patients with acute zonal occult outer retinopathy. Retina 37: 1104-1119, 2017. 
10. Ghasemi Falavarjani K, Wang K, Khadamy J and Sadda SR: Ultra-wide-field imaging in diabetic retinopathy; an overview. J Curr Ophthalmol 28: 57-60, 2016.

11. Bernardes R, Serranho P and Lobo C: Digital ocular fundus imaging: A review. Ophthalmologica 226: 161-181, 2011.

12. Bae K, Cho K, Kang SW, Kim SJ and Kim JM: Peripheral reticular pigmentary degeneration and choroidal vascular insufficiency, studied by ultra wide-field fluorescein angiography. PLoS One 12: e0170526, 2017.

13. Zhou J and Wei W: Optos 200Tx scanning laser ophthalmoscope for wide-field fluorescein angiography and its clinical application. Int Rev Ophthalmol 2: 103-106, 2015.

14. Witmer MT, Parlitsis G, Patel S and Kiss S: Comparison of ultra-widefield fluorescein angiography with the Heidelberg Spectralis(®) noncontact ultra-widefield module versus the Optos(®) Optomap(®). Clin Ophthalmol 7: 389-394, 2013.

15. Kernt $M$ and Kampik A: Imaging of the peripheral retina. Oman J Ophthalmol 6 (Suppl 1): S32-S35, 2013.

16. Wang K, Ghasemi Falavarjani K, Nittala MG, Sagong M, Wykoff CC, van Hemert J, Ip M and Sadda SR: Ultra-wide-field fluorescein angiography-guided normalization of ischemic index calculation in eyes with retinal vein occlusion. Invest Ophthalmol Vis Sci 59: 3278-3285, 2018
17. Chhablani $\mathrm{J}$ and Jayadev C: Future in retinal imaging for clinicians. Indian J Ophthalmol 63: 370-372, 2015.

18. Widmer LK, Biland L, Delley A and da Silva A: The importance of peripheral-arterial occlusive diseases in medical practice. Conclusions from the Basel study. Schweiz Med Wochenschr 113: 1824-1827, 1983 (In German).

19. Tan CS, Chew MC, Lim LW and Sadda SR: Advances in retinal imaging for diabetic retinopathy and diabetic macular edema. Indian J Ophthalmol 64: 76-83, 2016.

20. Gelfand JM, Nolan R, Schwartz DM, Graves J and Green AJ: Microcystic macular oedema in multiple sclerosis is associated with disease severity. Brain 135: 1786-1793, 2012.

21. Espina MP, Arcinue CA, Ma F, Camacho N, Bartsch DU and Freeman WR: Analysis of a confocal scanning laser ophthalmoscope noncontact ultra-wide field lens system in retinal and choroidal disease. Retina 35: 2664-2668, 2015.

22. Kida T: Mystery of retinal vein occlusion: Vasoactivity of the vein and possible involvement of endothelin-1. BioMed Res Int 2017: 4816527, 2017.

This work is licensed under a Creative Commons Attribution-NonCommercial-NoDerivatives 4.0 International (CC BY-NC-ND 4.0) License. 